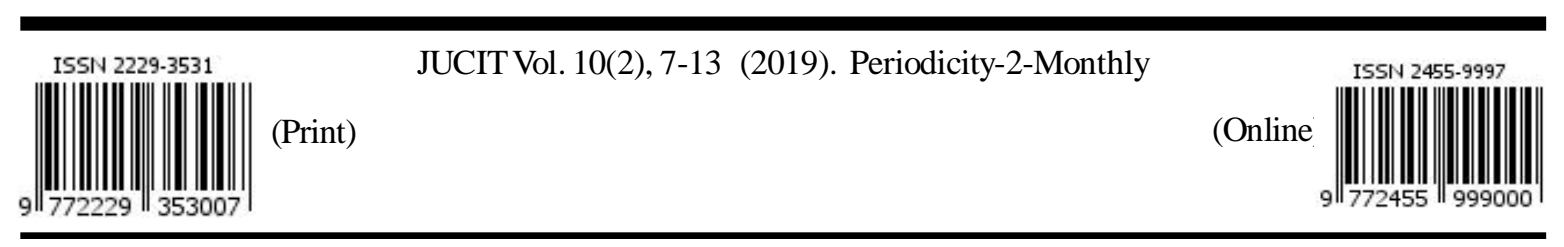

JOURNAL OF ULTRA COMPUTER \& INFORMATION TECHNOLOGY
An International Open Free Access Peer Reviewed Research Journal of Computer
Science Engineering \& Information Technology
website:- www.compitjournal.org

\title{
Energy Efficient Routing Protocols for Wireless Sensor Networks
}

\author{
SWIKRITI DANDOTIA $^{* 1}$ and M. A. RIZVI ${ }^{2}$ \\ ${ }^{* 1}$ M.tech Scholar Computer engineering and applications NITTTR, Bhopal (India) \\ ${ }^{2}$ Associate Professor and Head Computer engineering and applications NITTTR, Bhopal (India) \\ Corresponding Author Email:- marizvi@nitttrbpl.ac.in \\ http://dx.doi.org/10.22147/jucit/100201
}

Acceptance Date 26th March, 2019, Online Publication Date 2nd April, 2019

\begin{abstract}
Efficient design and implementation of wireless sensor networks has become a hot area of research in recent years, due to the vast potential of sensor networks to enable applications that connect the physical world to the virtual world. Wireless sensor networks sense and collect data from a certain area, process and transmit them to the sink. If the distance of communication is large, messages are to be propagated through intermediate nodes. The main emphasis of this work is to find out and overcome limitations of the wireless sensor networks such as: limited energy resources, varying energy consumption based on location, high cost of transmission, and limited processing capabilities. The algorithms are mainly based on location, power levels, and load on the node, and their goal is to achieve better target sensing with minimizing the power consumption and maintenance throughout the network so that the majority of the nodes consume their power supply at relatively the same rate regardless of physical location. Different metrics will be used to calculate results employing some fixed iterations, and therefore an optimal solution can be formed throughout the simulation. A number of network topologies may be simulated with a pre defined set of parameters for optimal results. We have simulated some energy efficient protocols namely GRP (Geographic Routing Protocol), TORA (Temporally ordered routing algorithm), AODV (Adhoc on Demand Distance Vector Routing), OLSR (Optimized Link State Routing Protocol) and DREAM (Distance Routing Effect Algorithm for Mobility) and have analyzed the results on 6(six) various metrics.

Introduction to Wireless Sensor Networks

Efficient design and implementation of wireless sensor networks has become a hot area of research in recent years, due to the vast potential of sensor networks to enable applications that connect the physical world to the virtual world. In the coming years, as advances in micro-fabrication technology allow the cost of manufacturing sensor nodes to continue to drop, increasing deployments of wireless sensor networks are expected, with the networks eventually

growing to large numbers of nodes (e.g., thousands) figure 1-1 Potential applications for such large-scale wireless sensor networks exist in a variety of fields, including medical monitoring, environmental monitoring, surveillance, home security, military operations, and industrial machine monitoring.

Recent advances in micro-electro-mechanical systems $\left(\mathrm{MEMS}^{2}\right)$ technology, wireless communications, and digital electronics have enabled the development of lowcost, low-power, multifunctional sensor nodes that are small
\end{abstract}

This is an open access article under the CC BY-NC-ND license (https://creativecommons.org/licenses/by-nc-sa/4.0) 
in size and communicate untethered in short distances. These tiny sensor nodes, which consist of sensing, data processing, and communicating components, leverage the idea of sensor networks based on collaborative effort of a large number of nodes ${ }^{1}$. Unlike a centralized system, the node of a wireless sensor network is subject to a unique set of resource constraints, such as finite on-board battery power and limited network communication bandwidth ${ }^{2}$.

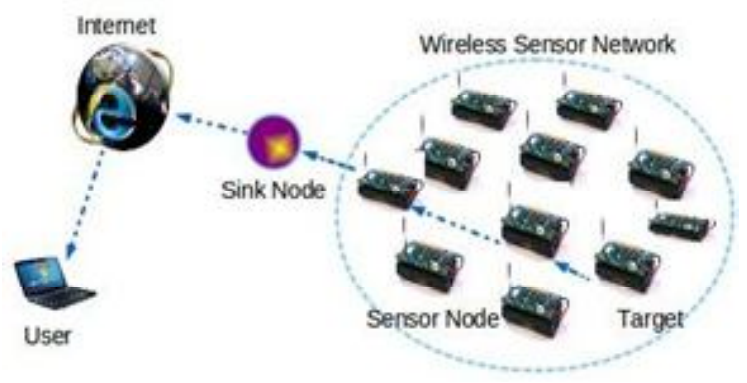

Figure 1-1 Communication architecture of a wireless sensor network ${ }^{1}$

\subsection{Architecture of Wireless Sensor Networks :}

This work explores the WSN architecture according to the OSI model with some protocols in order to achieve good background on the wireless sensor networks and help readers to find a summary for ideas, protocols and problems towards an appropriate design model for $\mathrm{WSNs}^{3}$. Figure 1-2 shows the general architecture of the WSN. Sensor nodes make up a sensor field sense the data and route it to a sink. The sink is a special sensor node connected to a personal computer, which is capable of transferring the data over the internet ${ }^{4}$

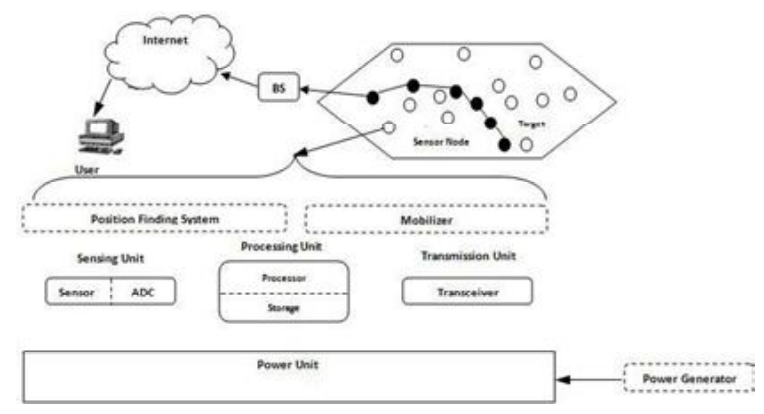

Figure 1-2 General Architecture of Wireless Sensor Networks

\subsection{Methodology :}

i) Different metrics will be used to calculate results employing some fixed iterations, and therefore an optimal solution can be formed throughout the simulation.

ii) A no. of network topologies may be simulated with a pre defined set of parameters for optimal results.

iii) Four routing protocols namely AODV, TORA, OLSR and DREAM have been selected from different topologies and designs for purpose of comparative analysis

iv) All these routing protocols will be tested on a self-written General Purpose Simulator OPNET.

\subsection{Implementation of Protocols on the OPNET Simulator:}

We have divided the entire research into 2 modules and 8 scenarios. In module 1 we have incorporated light traffic and in module 2 heavy traffic has been incorporated. We have the compared the simulation results of selected algorithms in reference to the light traffic and heavy traffic separately. The simulations were done on Opnet Modeller 17.5 Academic edition and the sensor networks used are wireless LAN workstations (mobile nodes) since they act same as that of any wireless sensor nodes. The description of the modules and their corresponding scenarios are given below.

\subsection{MODULE 1 Design :}

In this module we have made 4 scenarios and each scenario was run on same set of sensor nodes while the routing protocols were changed. In each scenario we made a wireless sensor network comprising of.

- 17 wireless sensor nodes (wireless LAN workstations).

- A wireless LAN server.

- Application configuration Object.

- Profile configuration Object.

- Mobility Configuration Object.

\subsubsection{Traffic incorporation in module 1:}

With the help of application and profile configuration objects we incorporated network traffic. A profile named PROF1 was created using profile configuration object which was configured to support only the ftp packets and with application configuration object, the application definition was set on ftp, so that the nodes could be configured for only ftp traffic. The sensor nodes will now be able to access the ftp traffic from the wireless LAN server. The paths followed by the sensor nodes to receive and send the traffic/packets were defined using the mobility configuration object. 


\section{A Scenario 1. AODV with light traffic:}

In this scenario we configured the wireless sensor nodes for light traffic with the help of application and profile configuration object, the server and the nodes were configured to run only the AODV routing protocol The network topology is given in the figure 4-1 below.

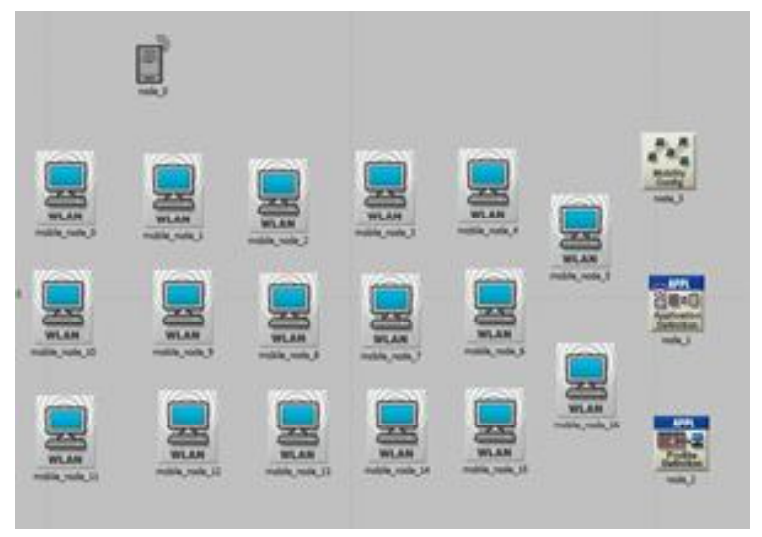

Figure 4-1 AODV with Light Traffic

\section{A. Scenario 2. TORA with light traffic:}

This scenario was configured using the wireless sensor nodes for light traffic with the help of application and profile configuration object, the wireless LAN server and the sensor nodes were configured to run only the TORA routing protocol. The network topology is given in the figure 4-2.

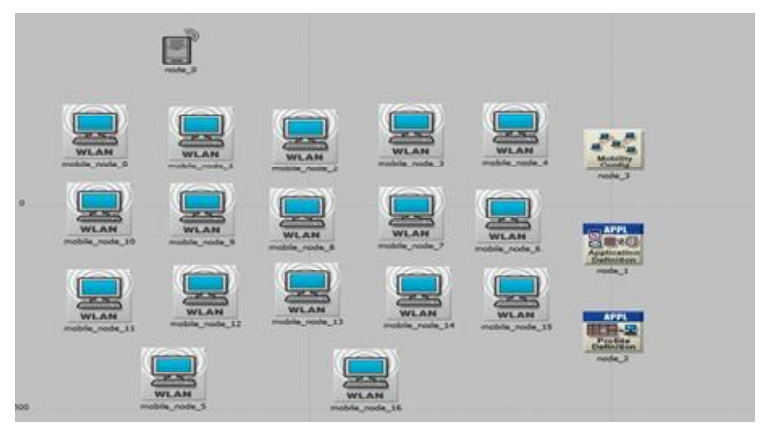

Figure 4-2 TORA with Light Traffic

\section{A. Scenario 3. DREAM with heavy traffic:}

This scenario was configured using the wireless sensor nodes for Heavy traffic with the help of application and profile configuration object, the wireless LAN server and the sensor nodes were configured to run only the DREAM routing protocol. The network topology is given in the figure 4-5.

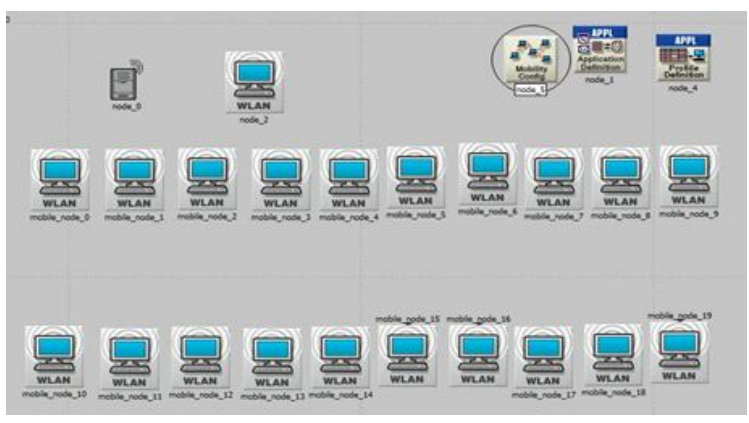

Figure 4-5. DREAM with heavy traffic

\section{B. Scenario 4. TORA with heavy traffic:}

In this scenario we configured the wireless sensor nodes for heavy traffic with the help of application and profile configuration object, the server and the nodes were configured to run only the TORA routing protocol. The network topology is given in the figure 4-6.

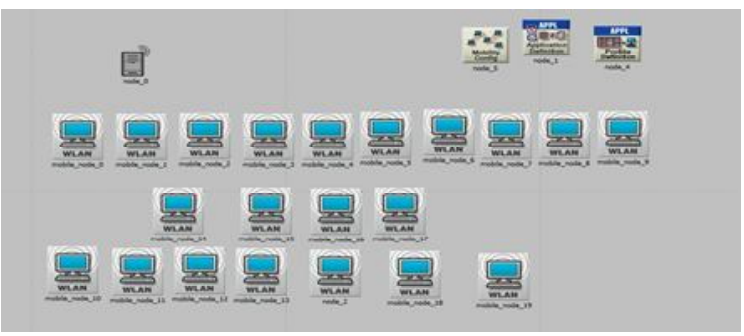

Figure 4-6 TORA with heavy traffic

\section{Scenario 5. AODV with heavy traffic :}

This Scenario was configured to allow only the AODV routing protocol to run on the individual sensor nodes. The application and profile configuration objects were set to allow heavy traffic as defined by the profile set. The jammer node was set to study the effect of jamming of some nodes on the whole network. The network topology is given in the figure 4-7

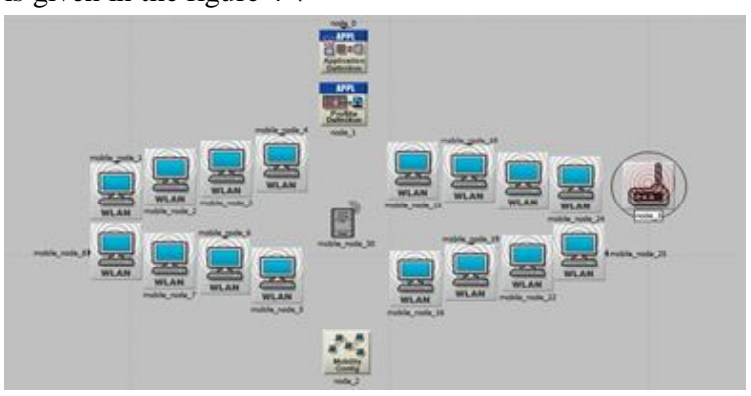

Figure 4-7 AODV with heavy Traffic 


\section{Simulations \\ MODULE 1}

\section{Phase $1^{\text {st }}$. Scenario 1 AODV}

\section{a. Load.}

Since we simulated the Wireless sensor network for 1 hour, the results displayed are more compact and precise for study. The graph below shows the load results on implementation of AODV routing protocol.

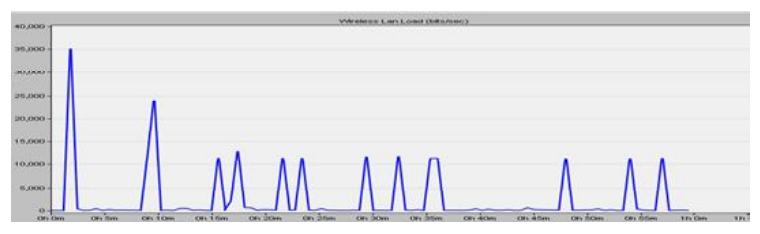

Figure 4-9. Load on AODV in bits/sec

From the graph it can be observed that at regular intervals the load keeps changing because the sensor node has to shift from one hop to another, and at times like at $18 \mathrm{~m}$ to $24 \mathrm{~m}$ it took a bit longer for the node to shift the next hop, this is because the node has to decide between the next hop. The load remains almost equal with some little fluctuations.

\section{a. Throughput:}

Node 6 in the wireless sensor network was selected as the reference node to study the throughput. The graph below shows the throughput on the reference node.

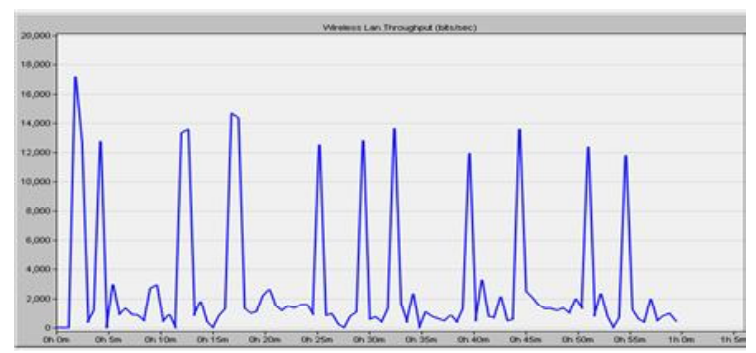

Figure 4-10 Throughput in AODV in bits/sec

The throughput remains almost constant at $2500 \mathrm{bits} / \mathrm{sec}$, with some fluctuations but these can be neglected.

\subsubsection{Module 1 Comparative Result Analysis:}

This module was divided into 4 different scenarios based on the routing protocol used; we implemented 4 routing protocols namely, AODV, TORA and GRP. All the four scenarios were compared on the parameters discussed earlier and the results were drawn out as discussed below.

\section{a. Throughput:}

The graph below shows the comparison of throughput for all the scenarios and the corresponding routing protocols. From the graph it can be observed that out of the four protocols OLSR has the maximum throughput at an average of $10000 \mathrm{bits} / \mathrm{sec}$. And the fluctuations are very little in OLSR and the graph runs smooth throughout the simulation. GRP protocol has an average throughput of almost 4000 bits/sec and so is the case with AODV and the lowest among the four is TORA with an average throughput of almost 2000 bits/sec.

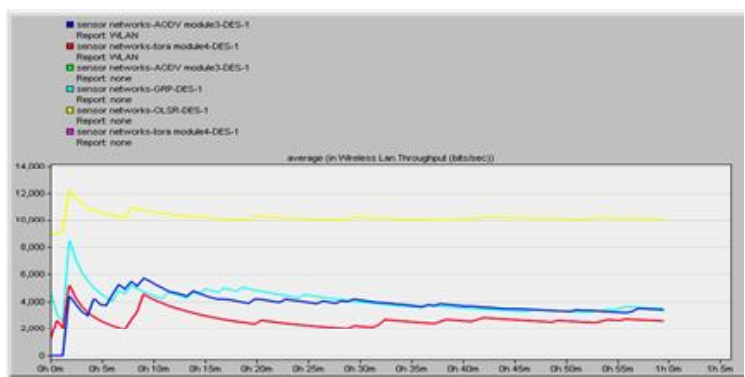

Figure 4-19 Comparison of Throughput of AODV-TORA-OLSR-GRP in bits/sec

Delay:

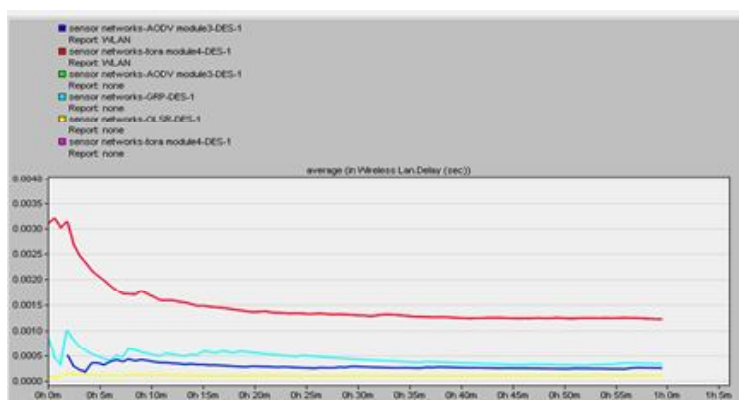

Figure 4-20 Comparison of Delay in AODV-TORAOLSR-GRP in seconds

From the study of the graph we find that among all the protocols used OLSR is the most efficient in terms of delay, the protocol has lowest delay among all the other protocols used, from the graph we can notice that the said protocols has almost $0 \%$ delay. TORA is having maximum delay of an average 0.0020 seconds. 


\section{b. Traffic Received:}

Below graph shows the comparison of module 1 with respect to selected parameter i.e., Data traffic received. From the study of the graph, it is clearly visible that at the beginning of the simulation there is a sharp increase in the traffic received by all the networks, the network with TORA implementation receives maximum traffic. But there is a shift from maximum value to a more constant trend of traffic received in all the networks. TORA remains constant at $60000 \mathrm{bits} / \mathrm{sec}$ followed by OLSR with a value of 50000 bits/sec. This shows the efficiency of TORA routing protocol in case of traffic received.

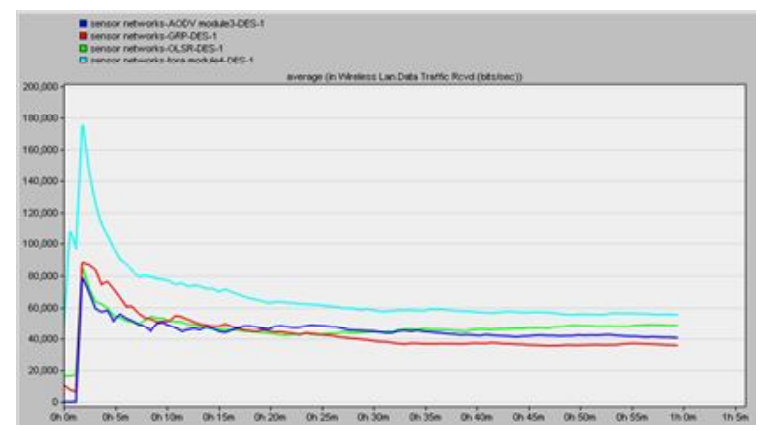

Figure 4-21 Comparison of Traffic Received in AODVTORA-OLSR-GRP in bits/sec

\section{c. Load:}

The graph below presents a comparison of module 1 on the selected parameter of Load (bits/sec). On an average load received by TORA is maximum than all the other routing protocols at an average value of $2000 \mathrm{bits} / \mathrm{sec}$. the load value shows the efficiency of TORA to withstand and carry a heavy traffic by easy and efficient way. The next efficient routing protocol is AODV with a value close to TORA at almost $1800 \mathrm{bits} / \mathrm{sec}$. The fluctuations in all the protocols are considerably negligible. OLSR protocol has also a comparably efficient load capacity.

\section{d. Media Access Delay:}

The graph below is the comparison on the basis of Media Access Delay in case of module 1.

From the study of the graph it can be noticed that in case of media access OLSR has the lowest delay, surpassing the other of its rivals in yet another parameter. TORA is the least efficient. Although media access delay is not $0 \%$ this time in case of OLSR but it's still less than the other routing protocols at a value of almost 0.0003 seconds.

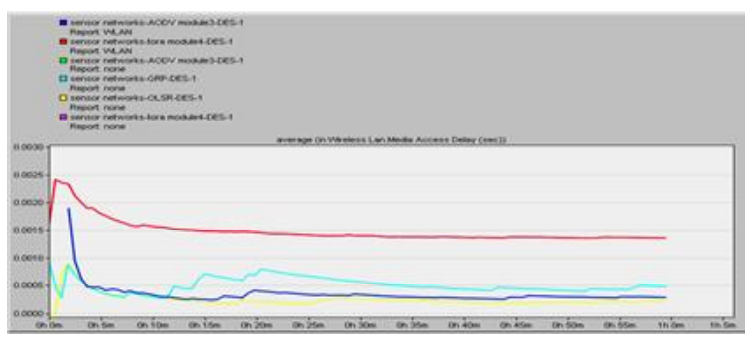

Figure 4-23 Comparison of Media Access Delay in AODV-TORA-OLSR-GRP in Seconds

\section{e. Retransmission Attempts:}

The graph below shows retransmission attempts made by networks in module and their comparison. The study shows the efficiency of TORA in case of retransmission attempts made. This shows that even though there are lots of connection drops in all the networks, TORA has tried to regain connections a maximum time. In the whole simulation of 1 hour it has made an average of 40-50 retransmissions.

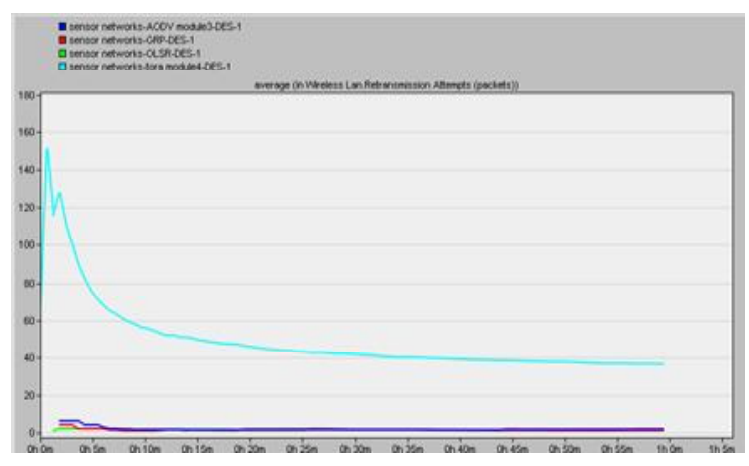

Figure 4-24 Comparison of Retransmission attempts in AODV-TORA-OLSR-GRP in packets

\subsubsection{Result Outcomes of Module 1:}

From the comparison of all the parameters of study we find that on most parameters we find that TORA is the efficient among all others, TORA is followed closely by OLSR and is the second most efficient routing protocols, in case of energy efficiency calculation we took two parameters of LOAD and THROUGHPUT and we concluded from the study of graphs obtained that TORA is most efficient of all, so our module 1 study reveal that out of the four protocols taken into consideration TORA turns out to be efficient in most of the parameters.

The below table shows the values obtained from the implementation of protocols for the module 1 at low Traffic. 
Table 4-1 Values of parameters on light traffic under simulation

\begin{tabular}{l|l|l|l|l}
\hline Parameter studied & $\begin{array}{l}\text { Values for } \\
\text { TORA }\end{array}$ & $\begin{array}{l}\text { Values for } \\
\text { OLSR }\end{array}$ & $\begin{array}{l}\text { Values for } \\
\text { AODV }\end{array}$ & $\begin{array}{l}\text { Values for } \\
\text { GRP }\end{array}$ \\
\hline Throughput(bits/sec) & 170,000 & 50,000 & 60,000 & 59,000 \\
Delay (seconds) & 0.0015 & 0.0001 & 0.0003 & 0.0004 \\
Load (bits/sec)Average & 90,000 & 72,000 & 68,000 & 64,000 \\
Re-Transmission attempts (average) & 40 & 6 & 3 & 2 \\
Data traffic Received (bits/sec) & 60,000 & 50,000 & 40,000 & 38,000 \\
Media access delay (seconds) & 0.0014 & 0.0003 & 0.0004 & 0.0005 \\
\hline
\end{tabular}

\section{MODULE 2:}

The table below has the values obtained from the implementation of protocols for the module 2 and the various parameters studied under high traffic.

Table 4-2 Values of parameters on heavy traffic under simulation

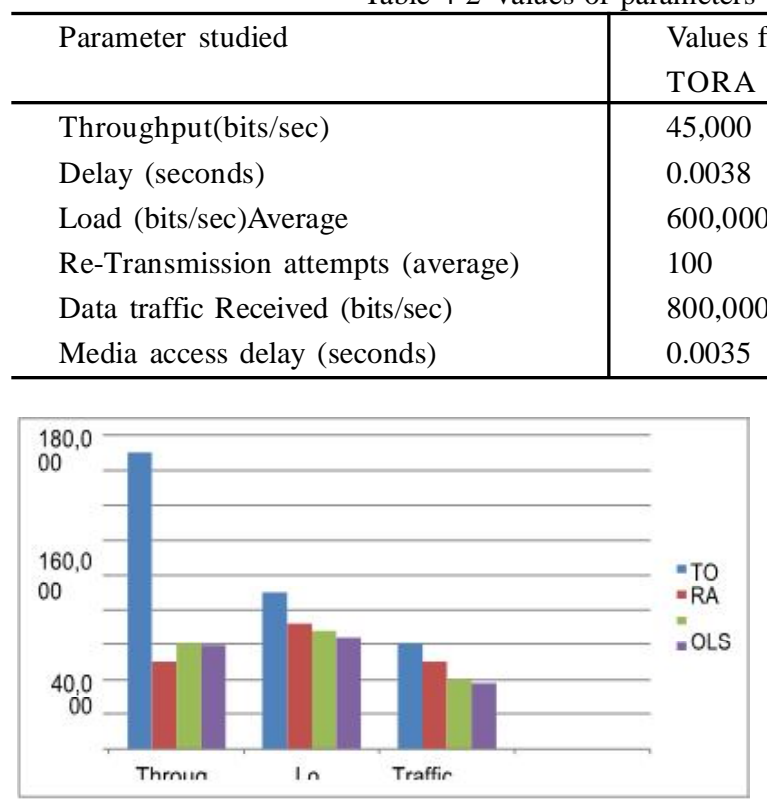

Figure 4-37 Comparison of parameters in bits/sec of TORA-OLSR-AODV-GRP under low traffic

The tables above have the values obtained for different parameters of study in our research, Table 4-1 has the values obtained for module 1 and table 4-2 has the values obtained for module 2. The graphs obtained after the evaluation of the values in module 1 show the dominance of TORA routing protocol and DREAM in module 2.

\subsection{Conclusion}

Since the energy resources of sensors are very limited and scarce, the main challenge in case of designing of

\begin{tabular}{l|l|l|l} 
& $\begin{array}{l}\text { Values for } \\
\text { OLSR }\end{array}$ & $\begin{array}{l}\text { Values for } \\
\text { AODV }\end{array}$ & $\begin{array}{l}\text { Values for } \\
\text { DREAM }\end{array}$ \\
\hline 40,000 & 40,000 & 60,000 \\
0.0015 & 0.0010 & 0.0045 \\
400,000 & 300,000 & 800,000 \\
20 & 40 & 80 \\
700,000 & 600,000 & $1,000,000$ \\
0.0025 & 0.0022 & 0.0060 \\
\hline
\end{tabular}

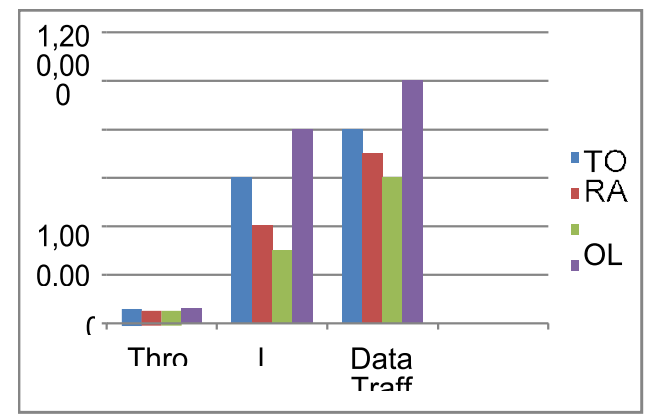

Figure 4-38 Comparison of parameters in bits/sec of TORA-OLSR-AODV-GRP under heavy traffic

a routing protocol for such devices is energy efficiency. In order to maintain the routes in the wireless sensor networks and to ensure a reliable multi-hop communication under the extreme limited conditions, we need routing protocols which are efficient in each and every spect. The routing should be hassle free and the data rate should be high, there should be less data loss and the data transmission on wireless links must be reliable. The delay in case of data reception and transmission must be extremely efficient. In case of routing in conventional and non-conventional wireless sensor networks there is a lot of dissimilarity. In wireless sensor 
networks routing differs from conventional routing in a number of ways.

$>$ The infrastructure is meager and less efficient.

$>$ There is unreliability of wireless links.

$>$ Chances of sensor node failure are high.

$>$ Strict energy saving requirement is to be met by routing protocols.

The main objective that must be kept in consideration while designing a routing protocol is that, it should to keep the sensor nodes operating for as longer as possible, thereby increasing the lifetime of the wireless sensor network. The main factor that determines the energy efficiency of a sensor node is dominated by the rate of data reception and transmission by it, so we may say that the design of a routing protocol should be in such a way that it should prolong the lifetime of a sensor node and so the wireless sensor network.

In our research, we studied a sample of five routing protocols by taking into account several parameters like, throughput, delay; media access delay, load, retransmission attempts made and data traffic received. We selected five sample routing protocols; DREAM, OLSR, GRP, TORA and AODV and analyzed the peculiarities of all these routing protocols, since all these routing protocols were designed to overcome the shortfalls of previously used routing protocols; the focus of our study was to select one among the chosen protocols which would qualify on maximum parameters of our study.

DREAM protocol in our case study gave a constant performance on most of the parameters. We studied the performance of the routing protocols for both heavy and light data traffic, TORA and OLSR showed a constant data flow in case of incorporation of light traffic and in most of the conditions TORA routing protocol was ahead in performance than other counter protocols in our study, but when the case was reversed and heavy traffic was incorporated, the protocol started to lose its sheen and began to fluctuate its results to a greater extent. But in case of both heavy and light data traffic incorporation the protocol that performed on a constant basis was DREAM, we concluded with DREAM that the throughput clearly increased, when the constant bit rate (CBR) was increased.

\section{References}

1. Akyildiz.I.F, Su. W, Sankarasubramaniam. Y, Cayirci, “Wireless Sensor Networks: A Survey”. Published in ELSEVIER, Computer Networks 38, 393-422 (2002).

2. Khemapech. I, Miller. A, Duncan.I, " Simulating Wireless Sensor Networks", School of computer Science University of St Andrews North Haugh, St Andrews.

3. A. Ahmad, A. Alhameed, B. Gurvinder, "Wireless Sensor Network Architecture", International Conference on Computer Networks and Communication Systems (CNCS 2012) IPCSIT vol.35(2012) (C) (2012) IACSIT Press, Singapore 11

4. Garg. KumKum, "Mobile Computing Theory and Practice", Pearson publications 2010. 